\title{
Forensic Sciences and Growth of Cardiology
}

Division of Cardiovascular Disease Biology, Rajiv Gandhi Center for Biotechnology, Trivandrum-695003, India, and Division of Cardiovascular Medicine, University of Arkansas, Little Rock, Arkansas, 72205, USA

Forensic sciences focussed on investigations of unnatural deaths and crimes have indisputably made significant contribution to advance medical and pharmaceutical sciences in addition to assisting the judiciary. Many examples can be cited of scientific discoveries of novel disorders, causes and mechanisms of diseases, drug toxicity, as well as effectiveness of therapeutic strategies that are secondary outcomes of medico-legal autopsies. Significant among them are related to cardiovascular sciences. Cardiology has notably had immense benefits from Forensic Sciences, early through autopsy studies and lately from forensic genetic testing.

The beginning of Cardiology is linked to discovery of circulation by William Harvey in the $16^{\text {th }}$ century. Harvey's discovery was made possible by the lift of ban on medical autopsies. Harvey was a pioneer in recognizing that clinic-pathologic correlations from postmortem findings are pivotal in gaining anatomical knowledge and recognizing the basis of clinical symptoms and signs. Andreas Vesalius, a predecessor of Harvey had noted marked differences between Galen's teaching and the real anatomy from observations in dissected human bodies. He traced the elaborate network of the blood vessels and accurately described the anatomy of the heart [1]. The significance of the approach of these two path finders is evident from the observations that over centuries, there has been no significant decrease in the rate of unexpected findings detected in either medico-legal autopsies or postmortem studies on patients dying from natural causes in hospitals $[2,3]$.

Autopsy studies have contributed immensely to our understanding of the anatomical basis of a number of heart diseases. A large number of heart diseases such as myocarditis, and carcinoid heart disease were first described during autopsies. Several infections that are not diagnosed clinically have been detected at autopsy for the first time as causes for cardiac symptoms and signs [4]. We recognize at present that influenza and pneumonia can trigger acute vascular events and increase the risk of myocardial infection. Studies have linked pre-eclampsia, a major cause of death due to vascular factors to maternal infection. It was through autopsy studies that Chaga's disease was recognized as a cause for cardiomyopathy as well as for sudden death in patients with asymptomatic infection with T. Cruzi $[5,6]$. Pathological features of another tropical problem rheumatic heart disease were clarified through post-mortem observations. Autopsy studies aided in understanding the pathogenesis of chronic valve disease, effects of mitral valve replacement and valve repair as well as the causes for failure of such treatment in patients with rheumatic valve disease.

Complex congenital heart diseases such as tetralogy of Fallot, transposition of the great vessels, tricuspid atresia, hypoplastic left heart syndrome, coarctation of the aorta, hypoplastic right heart syndrome, anomalous position of the heart, endocardial cushion defects and tricuspid valve dysplasias have all been characterized during postmortem examinations [7,8]. Post mortem studies on adults with congenital heart diseases and who have underwent surgical corrections could provide novel information and guidance for revision of present approaches to management of patients with congenital heart defects [9].

It was through autopsy studies that it was identified that fatal outcomes in coronary artery disease are a result of rupture of atherosclerotic plaques and superimposed coronary thrombosis.
Pathogenesis of atherosclerosis is also better understood now, thanks to a major study under the aegis of World Heart Federation. This multicentric research on Pathobiological Determinants of Atherosclerosis in Youth (PBDAY) had post-mortem material collected from those who had suffered fatal accidents and from 18 centres in 15 countries. This extensive investigation aided in identifying that the rate of atherosclerosis was higher in patients with high blood pressure, diabetes mellitus, smoking habits and high alcohol consumption. The study also revealed that atherosclerotic lesions develop early in life, independent of race, sex, geographical origin and that the progress to coronary heart disease is related to ethnic and life style factors [10].

Yet another significant contribution of forensic pathology has been in discerning the cause of sudden deaths. Autopsies have been diagnostic tools to establish the cause of death, and the missed diagnoses, if any, in instances where there has been no clinically identified heart problem. Sudden deaths due to inherited cardiac diseases in infants are difficult to diagnose because sudden death is the only first clinical manifestation. Such diseases are better appreciated currently thanks to post-mortem genetic analysis, a new tool to identify mutations in cardiac structural and functional proteins [11]. Cardiac channelopathies such as long-QT syndrome, Brugada syndrome, catecholaminergic polymorphic ventricular tachycardia, arrhythmogenic right ventricular cardiomyopathy/dysplasia and hypertrophic cardiomyopathy are conditions where genetic testing is reliable for clinical diagnosis [12]. Detection of cardiomyopathies and prediction of the risk of sudden death is now feasible by early screening of mutation carriers. Be that as it may, often the cause of sudden and unexpected death in those with normal hearts remains unexplained [13]. A large number of autopsy studies are in progress to discover clinically unrecognisable structural substrates and to elucidate the potential mechanisms of sudden cardiac death in the absence of any gross structural abnormality or heart defect. Post-mortem histology has provided evidence of concealed pathological substrates such as focal myocarditis in a few instances [14]. However, the cause of sudden cardiac death in young patients with histologically normal hearts remains a puzzle to be solved [15]. Research related to sudden cardiac death in infants is also in the stage of infancy and much progress has to be made for reliable clinical application [16].

Sudden natural deaths are also commonly associated with identifiable cardiac problems. One example is sudden deaths in patients with chronic heart failure. The Assessment of Treatment with Lisinopril and Survival (ATLAS) Trial evaluated the causes triggering sudden unexpected death in chronic heart failure, at autopsy. The study revealed that acute coronary events such as coronary thrombus, ruptured

${ }^{*}$ Corresponding author: Mehta JL, Division of Cardiovascular Medicine, UAMS and VAMC, Little Rock, USA, Tel: 501296 1426; E-mail: mehtajl@uams.edu

Received December 23, 2013; Accepted December 26, 2013; Published December 28, 2013

Citation: Kshemada K, Kartha CC, Mehta JL (2013) Forensic Sciences and Growth of Cardiology. J Forensic Res 5: e115. doi:10.4172/2157-7145.1000e115

Copyright: $\odot 2013 \mathrm{Kshemada} \mathrm{K}$, et al. This is an open-access article distributed under the terms of the Creative Commons Attribution License, which permits unrestricted use, distribution, and reproduction in any medium, provided the original author and source are credited. 
plaque, or myocardial infarction, usually not clinically diagnosed in patients with heart failure and associated coronary artery disease, were prevalent in such patients and might be important in triggering sudden death [17].

Post-mortem studies have also assisted in improving heart transplant procedures. The Necropsy Heart Transplantation study (NEHTS study) aimed to identify the discrepancies between clinical cause of death and autopsy findings in heart transplant recipients. The study reported that the main discrepancies between autopsy and clinical cause of death were errors in the diagnosis of causes of dysfunction in the transplanted organ, acute humoral rejection, cardiac allograft disease, pulmonary embolism and disseminated intravascular coagulation. The significance of this study is that major factors with potential for adverse impact in the survival of patients with heart transplant and clinically undiagnosed could be discovered [18]

Though rare, marijuana abuse can cause cardiac arrest with often fatal consequences [19]. Post-mortem toxicological analysis of patient's blood reveals the psychoactive secondary metabolite tetrahydrocannabinol (THC) in the blood of Cannabis sativus or marijuana abusers, who could die from cardiovascular events such as arrhythmias or myocardial infarction [20]. Cocaine is another psychoactive recreational drug which causes sudden death. Coronary obstructive lesions in patients with cocaine induced ischemic heart disease were found in an autopsy examination, to be related to coronary artery spasm, endothelial injury and platelet aggregation [21]. Autopsy studies on long term cocaine abusers revealed that the mechanisms of cocaine induced cardiac complications are coronary vasospasm associated with adventitial mast cell aggregates, coronary thrombosis, myocardial ischemia and lymphocytic myocarditis [22]. Techniques of forensic toxicology apart from tracing known recreational drugs such as cocaine, cannabis and amphetamine products are considered also useful to identify new substances with previously unknown potential for abuse. Forensic toxicology tools could be useful to investigate the cause of sudden unexpected cardiac deaths in teens with no history of heart disease and no or low risk for heart disease [23].

Valve repair, replacement, coronary arterial by-pass grafting and implantation of vascular stents and ventricular assist devices are common surgical procedures employed in the management of various heart diseases. Forensic pathology provides an opportunity to recognize short and long term complications that result from failure of the techniques or issues related to biocompatibility of the implant materials. Implants retrieved at autopsy can also be analysed to discern the causes for failure such as physical or chemical degradation. Such studies have been useful to redesign and develop novel arterial grafts, vascular stents and valves as well as alter techniques of implantation of such devices. Medico-legal autopsies are also sources for harvesting tissues for transplantation; an example is homograft valves for replacement in patients with valve defects. Post mortem analyses of homografts retrieved from patients who underwent valve implantation have aided to improve techniques for valve processing and reduce chances of immunologic rejection and valve degeneration. Forensic specialists have led the way to regulate the legal framework necessary for harvesting hearts for transplantation.

Many pharmaceutical drugs, though validated in clinical trials before widespread clinical use, often have adverse effects, some fatal [24]. Pharmaceutical forensics is a specialty which investigates the causes for such adverse effects using samples collected post-mortem and the data from such research are used to modify the material characteristics of drug and improve manufacturing processes. Iatrogenic effects which account for a large number of deaths from cardiac complications can also be identified during autopsy examinations. For example cardiac hypertrophy resulting from the cardiotropic effect of anabolic androgenic steroids was identified only by autopsy studies [25]. Forensic pathologists have contributed to the development of several drugs used for the treatment of coronary artery disease including antithrombotic drugs.

Cardiotoxicity of some of the poisonous plants and the mechanism of cardiac toxicity have been understood thanks to forensic analysis. Medicinal plants such as Cerebera odollum, Atropa belladonna, Datura fastuosa, Colchicum autumnale have been thus recognized to have toxic effects $[26,27]$. The toxic dose causing fatality has also been identified by post-mortem studies [28].

In summary, there has been a mutually beneficial association among cardiologists, cardiovascular scientists and forensic pathologists and scientists. Medico-legal autopsies have revealed unexpected yet clinically important cardiac lesions. The findings have been of significant help to improve quality of patient care and advance cardiology practice. Different branches of forensic science such as forensic medicine, forensic pathology, forensic pharmacology, forensic toxicology and forensic genetics have contributed to the growth of cardiovascular disease biology and invention of novel strategies for treatment of patients with heart diseases. Certainly much more can be expected from this collaboration.

\section{References}

1. Gulczyński J, Iżycka-Świeszewska E, Grzybiak M (2010) Short history of the autopsy: Part II From the second half of the $16^{\text {th }}$ century to contemporary times. Pol J Pathol 3: 169-175.

2. Goldman L, Sayson R, Robbins S, Cohn LH, Bettmann M, et al. (1983) The value of the autopsy in three medical eras. N Engl J Med 308: 1000-1005.

3. Hickling MF, Pontefract DE, Gallagher PJ, Livesey SA (2007) Post mortem examinations after cardiac surgery. Heart 93: 761-765.

4. Smeeth L, Casas JP, Hingorani AD (2007) The role of infection in cardiovascular disease: more support but many questions remain. Eur Heart J 28: 1178-1179.

5. Gurgel CB, Ferreira MC, Mendes CR, Coutinho E, Favoritto P, et al. (2010) Apical lesions in Chagas' heart disease patients: an autopsy study. Rev Soc Bras Med Trop 43: 709-12.

6. Satoh F, Tachibana H, Hasegawa I, Osawa M (2010) Sudden death caused by chronic Chagas disease in a non-endemic country: Autopsy report. Pathol Int 60: $235-240$.

7. Kay MH, Moodie DS, Sterba R, Murphy DJ Jr, Rosenkranz E, et al.(1991) The value of the autopsy in congenital heart disease. Clin Pediatr (Phila) 30: 450454.

8. Lagarde O, Garabedian V, Coignard A, Duret JC, Piwnica A, et al. (1980) Congenital tricuspid insufficiency due to valvular dysplasia. Review of the literature in light of a case in a 40-year-old adult. Arch Mal Coeur Vaiss. 73: 387-396.

9. Goetzová J, Benesová D (1981) Congenital heart diseases at autopsy of stillborn and deceased children in the Central Bohemian Region. Cor Vasa 23 8-13.

10. Lesauskaite V, Tanganelli P, Bianciardi G, Simoes C, Toti P, et al. (1999) World Health Organization (WHO) and the World Heart Federation (WHF) Pathobiological Determinants of Atherosclerosis in Youth (PBDAY) Study. Histomorphometric investigation of the aorta and coronary arteries in young people from different geographical locations. Nutr Metab Cardiovasc Dis 9 : 266-76.

11. Tester DJ, Ackerman MJ (2009) Cardiomyopathic and channelopathic causes of sudden unexplained death in infants and children. Annu Rev Med 60: 69-84.

12. Bai R, Napolitano C, Bloise R, Monteforte N, Priori SG (2009) Yield of genetic screening in inherited cardiac channelopathies: how to prioritize access to genetic testing. Circ Arrhythm Electrophysiol 1: 6-15. 
Citation: Kshemada K, Kartha CC, Mehta JL (2013) Forensic Sciences and Growth of Cardiology. J Forensic Res 5: e115. doi:10.4172/2157$7145.1000 \mathrm{e} 115$

Page 3 of 3

13. Basso C, Carturan E, Pilichou K, Rizzo S, Corrado D, et al. (2010) Sudden cardiac death with normal heart: molecular autopsy. Cardiovasc Pathol 19: 321 325 .

14. Chugh SS, Kelly KL, Titus JL (2000) Sudden cardiac death with apparently normal heart. Circulation 102: 649-654.

15. Corrado D, Basso C, Thiene G (2001) Sudden cardiac death in young people with apparently normal heart. Cardiovasc Res 50: 399-408.

16. Campuzano O, Beltrán-Alvarez P, Iglesias A, Scornik F, Pérez G, et al.(2010) Genetics and cardiac channelopathies. Genetics in Med 12: 260-267.

17. Uretsky BF, Thygesen K, Armstrong PW, Cleland JG, Horowitz JD, et al.(2000) Acute Coronary Findings at Autopsy in Heart Failure Patients with Sudden Death Results From the Assessment of Treatment With Lisinopril and Survival (ATLAS) Trial. Circulation 102: 611-616.

18. Bocchi EA, Valette TN (2012) Clinical Diagnoses and Autopsy Findings After Heart Transplantation: Discrepancies Affecting Management And Survival NEHTS Study (Necropsy Heart Transplantation Study). J Am Coll Cardiol 59: E929-E929.

19. Lindsay AC, Foale RA, Warren O, Henry JA (2005) Cannabis as a precipitant of cardiovascular emergencies. Int J Cardiol 104: 230-232.

20. Aryana A, Williams MA (2007) Marijuana as a trigger of cardiovascular events: Speculation or scientific certainty? Int J Cardiol 118: 141-144.
21. Kolodgie FD, Virmani R, Cornhill JF, Herderick EE, Smialek J (1991) Increase in atherosclerosis and adventitial mast cells in cocaine abusers: an alternative mechanism of cocaine-associated coronary vasospasm and thrombosis. J Am Coll Cardiol 17: 1553-1560.

22. Simpson RW, Edwards WD (1986) Pathogenesis of cocaine-induced ischemic heart disease. Autopsy findings in a 21-year-old man. Arch Pathol Lab Med 110: 479-484.

23. Bachs L, Mørland $H$ (2001) Acute cardiovascular fatalities following cannabis use. Forensic Sci Int 124: 200-203.

24. Buajordet I, Ebbesen J, Erikssen J, Brørs O, Hilberg T (2001) Fatal adverse drug events: the paradox of drug treatment. J Intern Med 250 : 327-341.

25. Far HR, Agren G, Thiblin I (2012) Cardiac hypertrophy in deceased users of anabolic androgenic steroids: an investigation of autopsy findings. Cardiovasc Pathol 21: 312-316.

26. Ballantyne B, Mars TC, Tumer P (1995) Fundamentals of toxicology, General and Applied toxicology. McMillan Press.

27. Singh D, Jit I, Tyagi S (1999) Changing trends in acute poisoning in Chandigarh zone: A 25 year autopsy experience from a tertiary care hospital in northern India. Amer j Forensic Pathol 20: 203-210.

28. Khajja BS, Sharma M, Singh R, Mathur GK (2011) Forensic Study of Indian Toxicological Plants as Botanical Weapon (BW): A Review. J Environment Analytic Toxicol 1: 112 